\title{
Accuracy of endoscopic ultrasound-guided biopsy of focal liver lesions
}

\author{
Vlad Andrei Ichim¹, Romeo Ioan Chira ${ }^{1,2}$, Petru Adrian Mircea1, Georgiana Anca Nagy ${ }^{1,2}$, \\ Doinita Crisan $^{3}$, Mihai Adrian Socaciu ${ }^{4}$
}

${ }^{1} 1^{\text {st }}$ Department of Internal Medicine, "Iuliu Hatieganu" University of Medicine and Pharmacy, ${ }^{2}$ Emergency County Hospital Cluj, $1^{\text {st }}$ Medical Clinic, Gastroenterology Department, ${ }^{3}$ Emergency County Hospital Cluj, Department of

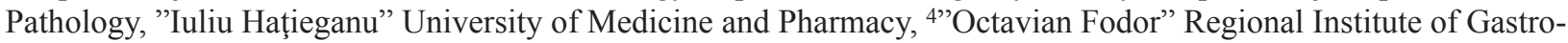
enterology and Hepatology, Department of Radiology, "Iuliu Hatieganu" University of Medicine and Pharmacy, Cluj-Napoca, Romania

\begin{abstract}
Aim: Endoscopic ultrasound (EUS) has become an indispensable method for diagnosis in gastroenterology and new indications for EUS continue to emerge. However, there are limited data regarding the accuracy of EUS-guided biopsy of hepatic focal lesions. The aim of this study was to assess the diagnostic yield of EUS-guided fine needle aspiration (FNA) of focal liver lesions. Material and methods: We conducted a prospective study in which patients with focal liver lesions, detected by transabdominal ultrasound and computed tomography or magnetic resonance imaging, underwent EUS-guided FNA to determine the diagnostic yield of the procedure. Results: In 47/48 of patients, the results of EUS-FNA were positive for malignancy, while in one case the acquired fragment was insufficient for appropriate histological analysis. Diagnostic yield was 0.98 . In $83 \%$ of the cases biopsies were taken from the left lobe and in $17 \%$ from the right lobe with the same technical success rate. The most common diagnosis was metastatic adenocarcinoma of the pancreas ( $26 \%$ cases) followed by cholangiocarcinoma (17\% cases). Concurrent sampling of other sites in addition to the liver and/or primary tumor was realized in $35 \%$ of the cases, with results that correlated with the liver biopsy and with the primary tumor biopsy. We reported no immediate or long-term complications in any of the patients. Conclusions: EUS guided fine needle aspiration/biopsy of focal liver lesions is safe, provides a very high diagnostic accuracy and should not be considered only as a rescue method after failure of percutaneous guided biopsies.
\end{abstract}

Keywords: endoscopic ultrasound-fine needle aspiration (EUS-FNA); liver biopsy; focal liver lesions

\section{Introduction}

Endoscopic ultrasonography (EUS) has become an indispensable diagnostic method in gastroenterology and

Received 25.09.2019 Accepted 27.12.2019

Med Ultrason

2020, Vol. 22, No 1, 20-25

Corresponding author: Romeo I. Chira, MD, PhD

$1^{\text {st }}$ Medical Clinic, Gastroenterology Department

3-5 Clinicilor street,

400006, Cluj Napoca, Romania

Phone: +40 751130280

E-mail: romeochira@yahoo.com new indications for EUS continue to emerge [1]. There has been a sustained progress in EUS diagnostic, potential due to constantly improving imaging systems and needles, which resulted in better tissue acquisition and lower technical failure rate [2-7].

Ultrasonography (US) or computed tomography (CT) guided liver biopsy are frequently used for the diagnosis of focal liver lesions. Although both methods have a high diagnostic accuracy of approximately $90 \%$ [8-13], in certain patients, US or CT guidance may be impossible because of suboptimal visualization of small liver lesions or the lesions are difficult to be differentiated from the normal parenchyma. 
The usefulness of EUS guided fine needle aspiration (EUS-FNA) of focal liver lesions has also been investigated, and it has a similar diagnostic yield, ranging from $80-100 \%$ [14]. However, EUS has several advantages over other imaging modalities regarding optimal visualization of the liver, availability without regard to patient body type, presence of massive ascites, lesions in the caudate lobe or non-cooperating patients. These advantages combined with limited adverse events, makes EUS an excellent modality for diagnosis and staging of malignant conditions concerning the liver. Still, there are a limited number of papers that analyze the feasibility and safety of EUS guided FNA for focal liver lesions. EUS and FNA of liver lesions is considered a "rescue" diagnosis method, after an unsuccessful or impossible approach by classical biopsy guiding methods [14-16].

The objective of this study was to assess the diagnostic yield and safety of EUS-guided fine needle aspiration FNA of focal liver lesions and to discuss situations when to choose this procedure over the US/CT guided biopsy.

\section{Material and method}

\section{Patients}

We performed EUS-FNA for the following indications: to assess primary lesions and establish tissue diagnosis from the liver masses identified by US/CT/MRI, for pancreatic tumors with focal liver lesions, and in cases where due to poor accessibility it was difficult to obtain a tissue sample through percutaneous biopsy. In this prospective study, we evaluated 48 consecutive patients (21 females, average age of 66.3 years old, range 40-83 years old) who underwent EUS-FNA for the diagnosis of liver lesions in our institution, between August 2017 and March 2019. In all, but 2 cases, the primary tumor had been identified via imaging or endoscopy. The inclusion criteria were age over 18 years old, presence of solid liver masses revealed by US/CT/MRI that required biopsy (fig 1a). The exclusion criteria were hemostasis and bleeding disorders (international normalized ratio $>1.5$ [normal range $0.85-1.25$ ] or platelet count $<80000$ / $\mathrm{mm} 3$ [normal range $150000-450000 / \mathrm{mm}^{3}$ ]) and poor accessibility to the tumor by EUS. Written informed consent was obtained from all patients before the procedure. This study was approved by the local Ethics Committee.

\section{Endoscopic ultrasound-guided fine-needle biopsy procedure}

EUS-FNA was performed by a single expert endosonographer using a linear array echoendoscope (Olympus GF-UCT180 with ultrasound processor EU-ME 2). Tissue acquisition was performed with a $22 \mathrm{G}$ needle (Expect ${ }^{\circledR}$, Boston Scientific or EZ Shot $3+\AA$, Olympus Medical Systems).

Following the evaluation of the target lesion, including real-time Doppler US (fig 1b), FNA was performed either trans gastric for the left lobe (fig 1c) or transduodenal for the right lobe. The needle was advanced into the target lesion under EUS guidance. Once the lesion was punctured, the stylet was detached. The specimen was obtained by moving the needle back and forth within the lesion while applying negative pressure with a $10 \mathrm{~mL}$ syringe. Suction was released by closing the syringe lock and the needle was removed afterwards. Aspirated specimens were displaced by reinserting the stylet. The tissue sample was carefully inspected after each pass to determine the successful acquisition of specimen, with additional passes performed if necessary. The aspirate was then immersed in formalin for histology (fig 1d) and in some cases, when we procured a small amount of tissue laid out onto glass slides for cytology smears.

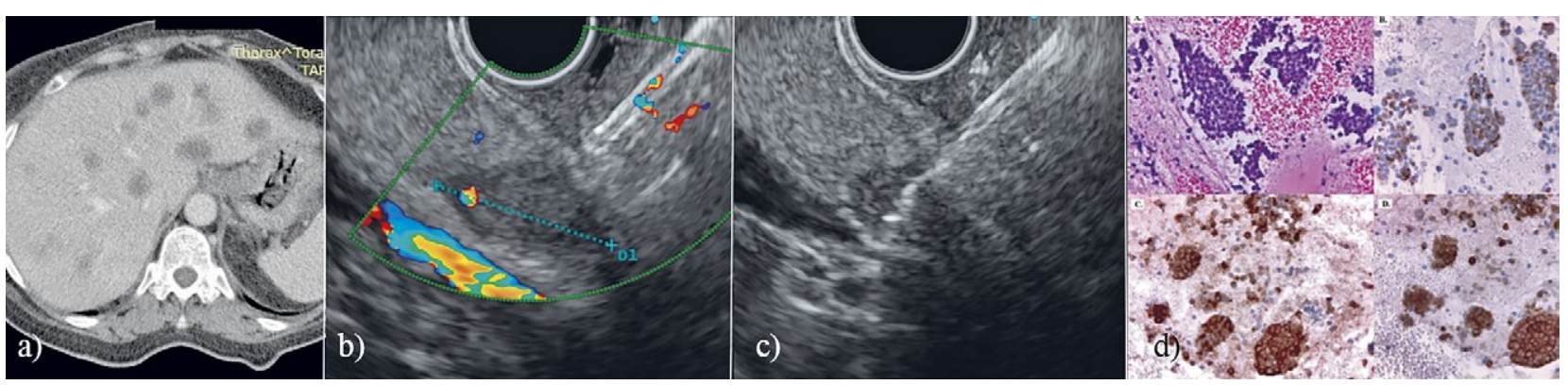

Fig 1. a) Axial contrast-enhanced computed tomography - liver metastasis of a neuroendocrine tumor; b) Endoscopic ultrasound (EUS) with color Doppler mode, used to exclude the presence of interposing vessels between transducer and target lesions; c) EUS fine needle aspiration (FNA) of the tumor in the caudate lobe, adjacent to the inferior vena cava; d) FNA - liver metastasis of a low grade neuroendocrine tumor. A. Nests of monotonous small cells, with scant cytoplasm and salt and pepper chromatin (Hematoxylin and Eosin stain, X 40). B. Tumor cells are positive for anti-cytokeratin AE1/AE3 antibody (X 40). C. Intense and diffuse staining of the cytoplasm with anti-chromogranin antibody (X 40). D. Tumor cells show strong cytoplasmic positivity for synaptophysin (X 40). 
If the patient presented an extrahepatic tumor presumed to be the primary one, or lesions in another organs of lymph nodes suspected to be metastasis, we performed FNA also from those tumors or suspected metastasis sites in the same session.

All patients were admitted and followed for complications for at least 2 days.

Immunohistochemistry staining was performed at pathologist's choice, after haematoxilin-eosin stains could not establish the diagnosis.

\section{Statistical analysis}

Nonparametric data are presented by mean and range. The diagnostic yield was calculated as the number of biopsies that resulted in a diagnosis divided by the total number of biopsies performed. To investigate whether there was a statistically significant difference between diagnostic accuracy of EUS-FNA of the left lobe and right lobe, we performed a categorical Chi-squared contingency analysis.

\section{Results}

In $47 / 48$ of patients, the results of EUS-FNA were positive for malignancy, while in one case the acquired fragment was inadequate. Diagnostic yield was 0.98 in our study.

The final diagnosis for the 48 liver biopsies is recorded in Table I.

\section{Characteristics of EUS examination}

Multiple liver lesions were found in $77 \%$ of the patients while $23 \%$ had a single lesion. Out of all biopsies, $83 \%$ were taken from the left lobe and $17 \%$ from the

Table I. Histopathological diagnosis of the hepatic lesions obtained after EUS-FNA.

\begin{tabular}{ll}
\hline Diagnosis & Number (\%) \\
\hline Metastatic liver lesions & \\
Pancreatic adenocarcinoma & $13(27)$ \\
Pulmonary adenocarcinoma & $4(8)$ \\
Mammary carcinoma & $3(6)$ \\
Neuroendocrine tumor & $3(6)$ \\
Colon adenocarcinoma & $3(6)$ \\
Gastric adenocarcinoma & $2(4)$ \\
Prostate adenocarcinoma & $2(4)$ \\
Small cell lung carcinoma & $1(5)$ \\
Endometrial carcinoma & $1(5)$ \\
Squamous cell carcinoma of the lung & $1(5)$ \\
Primitive liver malignancies & \\
Cholangiocarcinoma & $8(17)$ \\
Hepatocellular carcinoma & $6(13)$ \\
Non-diagnostic & $1(2)$ \\
Total & $48(100)$ \\
\hline
\end{tabular}

right lobe. Statistical analysis comparing the diagnostic accuracy of the right and left lobe lesions biopsy results provided no statistically significant difference $(\mathrm{p}=0.723)$.

We also performed EUS-FNA of the primary tumor in $27 / 48(56 \%)$ of patients while in $18 / 48(38 \%)$ cases it was not possible due to poor accessibility or high procedural risk and in $3(6 \%)$ cases the fragment was inadequate for diagnosis. In $35 \%$ of patients concurrent sampling of other sites in addition to the liver and/or primary tumor was performed. Thus, we performed EUS-FNA from lymph nodes (12 cases), adrenal gland (1 case), lung (1 case), prostate ( 1 case - by lower EUS), ascites ( 1 case) and vertebra ( 1 case). All the extrahepatic FNAs were positive for malignancy and the results correlated with the liver biopsy and with the primary tumor biopsy.

In addition to histology fragment immersed in formalin, cytology smears were examined in 5/48 patients, with a positive result for malignancy in $3 / 5$ cases and without elements of malignancy in $2 / 3$ cases. Immunohistochemistry staining was realized in $77 \%$ of cases, when haematoxilin-eosin stains could not establish the diagnosis.

The median number of passages was 2 (range: 1-4).

We reported no immediate or long-term complications.

\section{Discussion}

In our study EUS-guided focal liver lesions biopsy using $22 \mathrm{G}$ needle provided an excellent diagnostic yield $(0.98)$ with a small number of passes (median 2$)$. We also performed, in the same sessions, FNA from other sites (in 35\% of the cases) confirming malignancy in all cases.

Focal liver lesions are often discovered incidentally during an imaging test, such as US, CT or MRI. In other scenarios they are found in patients with risk factors for hepatic malignancy or during a preoperative staging of various malignancies. Detailed characterization of focal liver lesions represents an indispensable part of patient evaluation, as the presence or extent of liver involvement frequently changes the clinical stage and management.

In recent years, EUS-FNA has emerged as an alternative tool in the diagnosis and classification of liver lesions [14]. Most of the liver segments can be visualized with the echoendoscope and the proximity of the ultrasound probe to the liver provides high-quality images of the liver parenchyma, which may have a decisive role in the detection, characterization and histological diagnosis of liver lesions $[17,18]$. Therefore, the utility of EUS and EUS-guided FNA of the liver lies in the ability to detect and diagnose cancer, and, in some cases, upstage the disease and avoid surgery. 
There are a number of findings that highlight this study. The first is the overall excellent pathologic diagnostic rate of $98 \%$ obtained using this procedure. Previously published prospective studies with similar cohorts found diagnostic yields ranging from $82 \%$ to $94 \%$ [1927]. Secondly, in 17 out of the 33 patients with metastatic liver lesions, the biopsy from the primary tumor could not be performed. These were the cases with previous surgically removal of the primary tumor and now presented for late relapse or when the biopsy from the primary tumor was considered to have a high procedural risk. We had also 3 patients in which the primary tumor biopsy was non-diagnostic. In their cases, EUS-FNA had a significant impact, and they could avoid additional diagnostic procedures. Thirdly, our data demonstrate the versatility of the EUS approach. We diagnosed 13 different types of tumors, some of them not being traditionally diagnosed through EUS-FNA.

Concurrent biopsies of metastatic sites, other than the liver, during a single endoscopic procedure are another example of this versatility [28-30]. We were able to take a biopsy from the retroperitoneal lymph nodes, adrenal glands, lungs, vertebra and prostate. This procedure allows critical staging information to be obtained simultaneously when establishing a primary diagnosis, thus expediting the management of a group with difficult to diagnose neoplasms.

The cytological examination was performed in addition to the histology in $5 / 48$ cases. Out of those, 2 were inconclusive, while 3 had signs of malignancy, but could not be properly characterized. Cytology could have been useful if rapid on-site evaluation (ROSE) would have been available [31] and in cases where it was not possible to procure a histology specimen, and usually offers an incomplete diagnosis.

Anatomy limits the use of EUS FNA to the whole liver. The hilum, the left and proximal right lobe including the major portion of the intrahepatic biliary tract can be targeted by EUS. The caudate lobe masses can be also addressed and this is important as the percutaneous biopsy is challenging due to its anatomic location $[32,33]$. We had no case in which the biopsy could not be performed because of the location of the tumors, but this was probably related to the inclusion criteria. For example, we considered that patients with peripheral lesion in the right lobe could undergo percutaneous biopsy. Other elements must also be taken into consideration, such as contraindication to sedation or upper gastrointestinal tract stenosis. The cost of the EUS-FNA needles is higher than those of the percutaneous needles, therefore department resources and experience will also influence the decision, since there are no guidelines re- garding the first option when multiple techniques are viable.

The most common scenario we encountered in clinical practice is that of a patient diagnosed with focal liver lesions via CT/MRI/US referred to our Department for biopsy. In some cases, the primary tumor was not found or was more difficult to biopsy than the metastatic liver lesions. The decision to choose between EUS guided liver biopsy and percutaneous liver biopsy takes into consideration the size of the lesion (small lesions are more difficult to biopsy percutaneously than EUS guided), coagulation status (many patients with liver lesions have an altered coagulation status and EUS-FNA has a lower bleeding risk), and the need for multiple biopsies (e.g. pancreatic tumor with a focal liver lesion - biopsies can be performed from pancreas and liver at the same time).

In our study EUS-guided liver biopsy using 22G needle provided an excellent diagnostic yield with a minimal number of passes (median 2, range 1-4). Studies that compared accuracy depending on the needle size for EUS-FNA of the liver focal lesions are scarce $[34,35]$. $22 \mathrm{G}$ needles are preferred mainly due to greater flexibility and easier passage. Data is mixed regarding this aspect, and tissue adequacy seems to be higher for the $19 \mathrm{G}$ versus $22 \mathrm{G}$ needle because the latter one procures samples that are more prone to fragmentation during specimen processing [34,35]. However, it is still unclear if the diagnostic yield of the $19 \mathrm{G}$ needle is better, especially for focal liver lesions.

\section{Complications and safety}

EUS has a very good overall safety profile. Nonetheless, there are notable complications, esophageal perforation being the most serious of them [36]. However, the majority of complications related to EUS take place during EUS-FNA, and include infection, hemorrhage, bile peritonitis and exceptionally malignant seeding. A systematic review reported $0.02 \%$ mortality associated with EUS and EUS-FNA, while the overall rate of EUSFNA-specific morbidity was 0.98\% [37-39]. A 2019 systematic review on the subject of EUS-FNA of focal liver lesions found complication rates ranging from $0 \%$ (6/8 studies) to $4 \%$ in one study [14]. In that particular study, Berge et al reported complications in 6 patients (4\%) from which 1 death (suspected to have an occluded biliary stent at the time of EUS), 1 bleeding, 2 cases of post-procedural fever and 2 cases of post-procedural abdominal pain occurred [20]. In our study, there were no immediate or long term complications. To date, no occurrences of tumor seeding after EUS-FNA for liver metastases were reported [27]. 
Our study has some limits. First, it was a single-arm observational study and did not compare the outcomes of EUS-FNA with those of percutaneous US/CT guided biopsy. Therefore, further comparative studies with a large number of patients are necessary to confirm the superiority of EUS-FNA for liver lesions. Secondly, the limitations of EUS-FNA of the liver have to be underlined. The presence of vascular structures in the path of the needle may limit the access to the lesion. Nevertheless, with improving resolution, use of Doppler US, elastography [40], harmonic imaging and/or contrast enhancement [41] have improved the accuracy of the procedure.

\section{Conclusion}

The results of our study show that EUS-FNA of the focal liver lesions is a safe procedure, with high accuracy for the diagnosis of malignancy. Although EUS has not traditionally been used in the evaluation of the liver, EUS-FNA can be helpful for malignant liver mass diagnosis, especially in cases where the percutaneous approach is difficult.

\section{Conflicts of interest: none}

\section{References}

1. Saraireh HA, Bilal M, Singh S. Role of endoscopic ultrasound in liver disease: Where do we stand in 2017? World J Hepatol 2017;9:1013-1021.

2. Nieto J, Khaleel H, Challita Y, et al. EUS-guided fine-needle core liver biopsy using a novel 19-gauge needle with modified 1-pass, 1 actuation wet suction technique. Gastrointest Endosc 2018;87:469-475.

3. Shah ND, Sasatomi E, Baron TH. Endoscopic ultrasoundguided parenchymal liver biopsy: single center experience of a new dedicated core needle. Clin Gastroenterol Hepatol 2017;15:784-786.

4. Schulman AR, Thompson CC, Odze R, Chan WW, Ryou M. Optimizing EUS guided liver biopsy: comprehensive assessment of needle types and tissue acquisition techniques. Gastrointest Endosc 2017;85:419-426.

5. DiMaio CJ, Kolb JM, Benias PC, et al. Initial experience with a novel EUS-guided core biopsy needle (SharkCore): results of a large North American multicenter study. Endosc Int Open 2016;4:974-979.

6. Rodrigues-Pinto E, Jalaj S, Grimm IS, Baron TH. Impact of EUS-guided fine-needle biopsy with a new core needle on the need for on-site cytopathological assessment: A preliminary study. Gastrointest Endosc 2016;84:10401046.

7. Inoue T, Okumura F, Mizushima T, et al. Assessment of factors affecting the usefulness and diagnostic yield of core biopsy needles with a side hole in endoscopic ultrasoundguided fine-needle aspiration. Gut Liver 2016;10:51-57.
8. CaturelliE, Solmi L,Anti M, etal.Ultrasound guided fineneedle biopsy of early hepatocellular carcinoma complicating liver cirrhosis: a multicentre study. Gut 2004;53:1356-1362.

9. Swamy MC, Arathi C, Kodandaswamy C. Value of ultrasonography-guided fine needle aspiration cytology in the investigative sequence of hepatic lesions with an emphasis on hepatocellular carcinoma. J Cytol 2011;28:178-184.

10. Elsayes KM, Ellis JH, Elkhouly T, et al. Diagnostic yield of percutaneous image-guided tissue biopsy of focal hepatic lesions in cancer patients: ten percent are not metastases from the primary malignancy. Cancer 2011;117:4041-4048.

11. Francque SM, De Pauw FF, Van den Steen GH, Van Marck EA, Pelckmans PA, Michielsen PP. Biopsy of focal liver lesions: guidelines, comparison of techniques and costanalysis. Acta Gastroenterol Belg 2003;66:160-165.

12. Sainani NI, Schlett CL, Hahn PF, Gervais DA, Mueller PR, Arellano RS. Computed tomography-guided percutaneous biopsy of isoattenuating focal liver lesions. Abdom Imaging 2014;39:633-644.

13. Stattaus J, Kuehl H, Ladd S, et al. CT-guided biopsy of small liver lesions: visibility, artifacts, and corresponding diagnostic accuracy. Cardiovasc Intervent Radiol 2016;30:928-935.

14. Ichim VA, Chira RI, Mircea PA. Diagnostic yield of endoscopic ultrasound-guided biopsy of focal liver lesions. Med Pharm Rep 2019;92:15-20.

15. Chon HK, Yang HC, Choi KH, Kim TH. Endoscopic Ultrasound-guided liver biopsy using a core needle for hepatic solid mass. Clin Endosc 2019;52:340-346.

16. Shah ND, Baron TH. Endoscopic ultrasound and the liver: current applications and beyond. J Hepatobiliary Pancreat Sci 2018;25:171-180.

17. Magalhães J, Monteiro S, Xavier S, Leite S, de Castro FD, Cotter J. Endoscopic ultrasonography - emerging applications in hepatology. World J Gastrointest Endosc 2017;9:378-388.

18. Kim E, Telford JJ. Advances in endoscopic ultrasound, part 2: Therapy. Can J Gastroenterol 2009;23:691-698.

19. Nguyen P, Feng JC, Chang KJ. Endoscopic ultrasound (EUS) and EUS-guided fine-needle aspiration (FNA) of liver lesions. Gastrointest Endosc 1999;50:357-361.

20. tenBerge J, Hoffman BJ, Hawes RH, et al. EUS-guided fine needle aspiration of the liver: indications, yield, and safety based on an international survey of 167 cases. Gastrointest Endosc 2002;55:859-862.

21. DeWitt J, LeBlanc J, McHenry L, et al. Endoscopic ultrasound-guided fine needle aspiration cytology of solid liver lesions: a large single-center experience. Am J Gastroenterol 2003;98:1976-1981.

22. Hollerbach S, Reiser M, Topalidis T, König M, Schmiegel W. Diagnosis of hepatocellular carcinoma (HCC) in a highrisk patient by using transgastric EUS-guided fine-needle biopsy (EUS-FNA). Z Gastroenterol 2003;41:995-998.

23. McGrath K, Brody D, Luketich J, Khalid A. Detection of unsuspected left hepatic lobe metastases during EUS staging of cancer of the esophagus and cardia. Am J Gastroenterol 2006; 101:1742-1746. 
24. Crowe DR, Eloubeidi MA, Chhieng DC, Jhala NC, Jhala D, Eltoum IA. Fine-needle aspiration biopsy of hepatic lesions: computerized tomographic-guided versus endoscopic ultrasound-guided FNA. Cancer 2006;108:180-185.

25. Singh P, Mukhopadhyay P, Bhatt B, et al. Endoscopic ultrasound versus CT scan for detection of the metastases to the liver: results of a prospective comparative study. J Clin Gastroenterol 2009;43:367-373.

26. Lee YN, Moon JH, Kim HK, et al. Usefulness of endoscopic ultrasound-guided sampling using core biopsy needle as a percutaneous biopsy rescue for diagnosis of solid liver mass: Combined histological-cytological analysis. J Gastroenterol Hepatol 2015;30:1161-1166.

27. Oh D, Seo DW, Hong SM, et al. Endoscopic ultrasoundguided fine-needle aspiration can target right liver mass. Endoscopic Ultrasound 2017;6:109-115.

28. Anand D, Barroeta JE, Gupta PK, Kochman M, Baloch ZW. Endoscopic ultrasound guided fine needle aspiration of non-pancreatic lesions: an institutional experience. J Clin Pathol 2007;60:1254-1262.

29. Patel S, Jinjuvadia R, Devara A, et al. Performance characteristics of EUS-FNA biopsy for adrenal lesions: A metaanalysis. Endosc Ultrasound 2019;8:180-187.

30. Chira RI, Chira A, Ichim VA, et al. Endoscopic ultrasoundguided fine-needle aspiration (EUS-FNA) of paraesophageal lung tumors - diagnostic yield and added value. Med Ultrason 2019;21:377-381.

31. Selhi PK, Tyagi R, Bansal P, Kaur H, Sood N. Hepatic fineneedle aspiration cytology: The role of rapid on-site evaluation in the assessment of hepatic lesions. Turk J Gastroenterol 2018;29:442-447.

32. Awad SS, Fagan S, Abudayyeh S, Karim N, Berger DH, Ayub K. Preoperative evaluation of hepatic lesions for the staging of hepatocellular and metastatic liver carcinoma using endoscopic ultrasonography. Am J Surg 2002;184:601-604.
33. Keohane J, Dimaio CJ, Schattner MA, Gerdes H. EUSguided transgastric drainage of caudate lobe liver abscesses. J Interv Gastroenterol 2011;1:139-141.

34. Patel H, Rush N, DeWitt J, et al. P2285 - 22G VS. 19G needles for EUS guided transgastric for parenchymal disease: A comparative analysis. Program No. P2285. ACG 2018 Annual Scientific Meeting Abstracts. Philadelphia, Pennsylvania: American College of Gastroenterology. Available at: https://www.eventscribe.com/2018/ACG/ajaxcalls/PosterInfo.asp? efp=RFNSWFFHSFY2NDI0\&PosterID $=1616$ 29\&rnd $=0.5584448$

35. Mok SRS, Diehl DL, Johal AS, et al. Endoscopic ultrasound-guided biopsy in chronic liver disease: a randomized comparison of 19-G FNA and 22-G FNB needles. Endosc Int Open 2019;7:E62-E71.

36. Das A, Sivak MV Jr, Chak A. Cervical esophageal perforation during EUS: a national survey. Gastrointest Endosc 2001;53:599-602.

37. Jenssen C, Alvarez-Sánchez MV, Napoléon B, Faiss S. Diagnostic endoscopic ultrasonography: assessment of safety and prevention of complications. World J Gastroenterol 2012;18:4659-4676.

38. ASGE Standards of Practice Committee, Early DS, Acosta $\mathrm{RD}$, et al. Adverse events associated with EUS and EUS with FNA. Gastrointest Endosc 2013;77:839-843.

39. Wang KX, Ben QW, Jin ZD, et al. Assessment of morbidity and mortality associated with EUS-guided FNA: a systematic review. Gastrointest Endosc 2011;73:283290.

40. Conti CB, Cavalcoli F, Fraquelli M, Conte D, Massironi S. Ultrasound elastographic techniques in focal liver lesions. World J Gastroenterol 2016;22:2647-2656.

41. Hirooka Y, Itoh A, Kawashima H, et al. Contrast-enhanced endoscopic ultrasonography in digestive diseases. J Gastroenterol 2012;47:1063-1072. 